\title{
Rationaler Einsatz von Antiinfektiva: Erfahrungen mit Antibiotic Stewardship
}

Bernd Salzberger, Gabriele Birkenfeld, Verena Greifenberg, Frank Hanses,

Florian Hitzenbichler, Birgit Hobbhahn, Josef Köstler, Thomas Holzmann, Alexander Kratzer,

Ulrich Rothe, Barbara Schmidt, Wulf Schneider, Andre Gessner

\begin{abstract}
Die rasche Zunahme von Resistenzen ist ein weltweites Problem. Das Universitätsklinikum Regensburg etablierte im Jahr 2012 ein Antibiotic-StewardshipProgramm mit dem Ziel, die Qualität der Antiinfektivatherapie zu verbessern und damit langfristig den Trend der bakteriellen Resistenzentwicklung aufzuhalten.
\end{abstract}

\section{Einleitung}

\section{Antibiotikaeinsatz und Resistenz}

Antibiotikaresistenzen gegen viele humanpathogene Bakterien nehmen rasch zu ( $\bullet$ Abb. 1) $[1,2]$. Dieses Problem ist mittlerweile so relevant, dass es Thema bei mehreren politischen Gipfeltreffen $(G 7, G 8, G 20)$ und in der UN-Vollversammlung war, es gibt eine ausführliche präsidiale Stellungnahme hierzu aus den USA [3].

Diese Entwicklung ist nicht monokausal - aber eine Ursache ist der Einsatz von Antibiotika in der Humanmedizin. Antibiotika werden vorrangig zur Behandlung schwerer Infektionen, aber auch prophylaktisch und in vielen Situationen empirisch bereits beim Verdacht auf eine Infektion eingesetzt. Auch bei viralen Atemwegsinfektionen werden Antibiotika häufig verschrieben.

\section{Merke}

Schätzungen gehen davon aus, dass ca. $50 \%$ aller Gaben von Antibiotika in der Humanmedizin nicht indiziert bzw. nötig sind.

Der Zusammenhang zwischen der Intensität des Antibiotikaeinsatzes und der Entwicklung von Resistenzen bei Bakterien ist seit langem bekannt. Bereits 1945 hat Alexander Fleming, der Erfinder des Penicillins, auf die Gefahren der Resistenzentwicklung unter diesen Bedingungen beschrieben [4].

\section{Antibiotic Stewardship}

\section{Entwicklung}

Die Entwicklung neuer Antibiotika ist dringend nötig, wird aber nicht rasch zur Lösung der aktuellen Probleme beitragen können, da von der Identifizierung neuer Angriffspunkte bei Bakterien bis zum Einsatz in der Klinik viele Jahre vergehen.
Ein optimierter Einsatz von Antibiotika ist deshalb ein essenzieller Teil der Strategie gegen die Resistenzentwicklung. Der rationale (und damit auch zurückhaltende) Antibiotikaeinsatz vermeidet nicht nur Nebenwirkungen, sondern hat vermutlich auch das Potenzial, diese Entwicklung aufzuhalten oder zumindest zu verlangsamen. Und ferner könnte ein Umdenken oder eine Änderung der Gewohnheiten im Einsatz auch

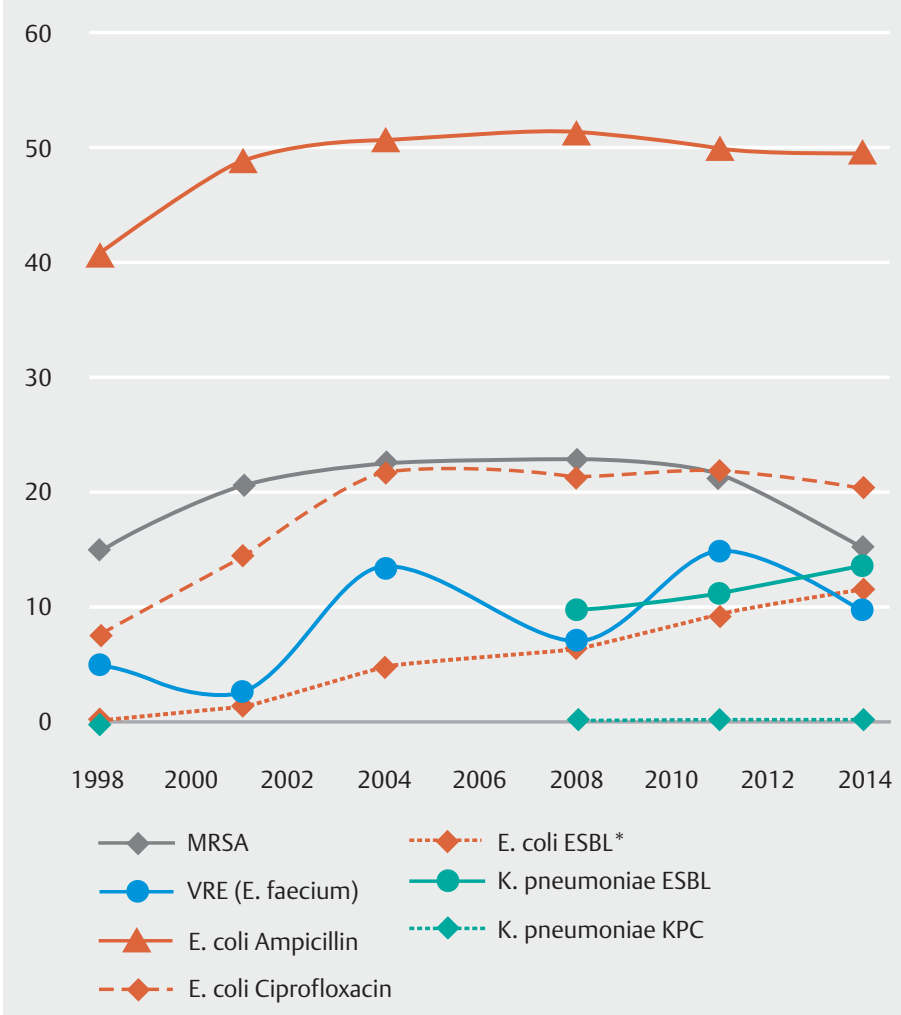

- Abb. 1 Entwicklung von Antibiotikaresistenzen in Deutschland (Daten 1998 - 2004 Paul-Ehrlich-Gesellschaft für Chemotherapie e. V., 2008 2014 ARS-Datenbank, Robert Koch-Institut) [1,2]. 


\section{INFOBOX}

Kernelemente eines Antibiotic-Stewardship-Programms nach CDC 2015 [9]:

- Leadership Commitment (Commitment und Unterstützung der Krankenhausleitung, ideell wie finanziell)

- Accountability (Verantwortung für das Programm liegt bei einem Spezialisten, am ehesten beim Infektionsmediziner)

- Drug Expertise (Einbeziehung eines spezialisierten Pharmazeuten/Apothekers, z. B. als stellvertretender Leiter)

- Action (Implementierung mindestens einer Intervention, z. B. des rechtzeitigen Absetzens von Antibiotika)

- Tracking (Monitoring des Antibiotikaeinsatzes und der Resistenzentwicklung)

- Reporting (Rückmeldung der Verbrauchs- und Resistenzdaten an Ärzte, Pflegepersonal und Klinikleitung)

- Education (regelmäßige Fortbildung bezüglich Antibiotikaresistenz und adäquaten Antibiotikaeinsatz)

langfristig das wertvolle Gut Antibiotika besser schützen [5].

Wie lässt sich dies erreichen? Es ist klar, dass der gut diagnostizierte, indizierte, gezielte, dosierte und auch wieder rechtzeitig beendete Einsatz von Antiinfektiva nicht einfach durch Vorschriften oder Gesetze erreicht werden kann. Der rasche Einsatz eines Antibiotikums kann in vielen klinischen Situationen lebensrettend sein - auch in der Prophylaxe. Regeln, die einen solchen Einsatz behindern, würde man zu Recht kaum akzeptieren und beachten [6]. Ansätze, die einen rationaleren Antibiotikaeinsatz erfolgreich erwirken sollen, müssen deshalb dieser Verantwortung gerecht werden und sollten von erfahrenen Ärzten geleitet werden. Solche Programme sind seit den 90er-Jahren überwiegend von Infektiologen, Mikrobiologen, Krankenhaushygienikern und Apothekern entwickelt worden.

In der englischsprachigen Literatur wurde der Begriff „Antibiotic Stewardship“ (ABS) bzw. „Antimicrobial Stewardship“ geprägt. Eine gute Übersetzung gibt es bisher nicht, der Begriff hat sich deshalb auch im Deutschen so durchgesetzt, obwohl „Steward“ und „Stewardship“ keineswegs selbsterklärend sind. Stewardship bedeutet in diesem Fall v.a. die Übernahme von Verantwortung. Ursprünglich wurde diese Bedeutung für die Organisation eines Haushalts gebraucht. Im vorliegenden Fall steht das Wohl der Patienten, aber auch das wertvolle Gut Antibiotika im Fokus.

Merke

Ziel eines ABS-Programms muss die Optimierung und Fokussierung von Antibiotikatherapien. Dies lässt sich durch eine Reihe verschiedener Maßnahmen erreichen, wie z. B. Fortbildungen, Erarbeitung von lokalen Leitlinien, Restriktion von Reserveantibiotika etc.

Ein wichtiger Teilaspekt ist dabei die Aus- und Bewertung der Maßnahmen und Rückmeldung der Ergebnisse an die Kliniker, die Antibiotika einsetzen. Der Aspekt, dass der Prozess aus Intervention, Messung und Bewertung der Ergebnisse und Feedback zur Justierung der Arbeit einen hohen Anteil von Management erfordert, wird in der englischsprachigen Literatur betont [7]. Die Arbeit und Ergebnisse solcher Programme sind in vielen Publikationen dargestellt und bewertet worden.

\section{FAZIT FÜR DIE PRAXIS}

In der Regel zeigt sich, dass sich eine Reduktion des Antibiotikaeinsatzes ohne negative Folgen für den Behandlungserfolg erreichen lässt - häufig auch mit einer Reduktion der Kosten [8].

Der Erfolg solcher Programme hat die Centers for Disease Control in den USA dazu veranlasst, Kernelemente daraus zu charakterisierten (s. Infobox) und diese Programme für alle Krankenhäuser zu empfehlen. In Deutschland ist man noch nicht ganz so weit: In der Deutschen Antibiotika-Resistenzstrategie (DART 2020) wird der Surveillance von Antibiotikaresistenzen und der Einsatz von Antibiotika breiter Raum gegeben. Antibiotic Stewardship wird zwar als wichtiges Feld der ärztlichen Aus-, Weiter- und Fortbildung aufgeführt, weitergehende Empfehlungen, z.B. zum Einsatz solcher Programme oder Maßnahmen in den Kliniken, sind hier jedoch nicht benannt $[9,10]$.

\section{Stand in Deutschland}

Eine ganze Reihe von ABS-Programmen ist auch in Deutschland gut etabliert, was nicht unerheblich zurückzuführen ist auf die Initiative einer vom Universitätsklinikum Freiburg etablierten und vom Bundesministerium für Gesundheit geförderten Fortbildungsreihe und des Netzwerks, das sich hierdurch gebildet hat. Dort wurden in intensiven interaktiven Kurse in mehreren Stufen Ärzte und Apotheker zu ABS-Experten geschult - mittlerweile sind mehr als 500 ABS-Experten ausgebildet. Die gleiche Gruppe hat eine S3-Leitlinie zum Aufbau von spezifischen Maßnahmen in einem solchen Programm erarbeitet und publiziert [11].

\section{ABS-Programm am UK Regensburg}

Am Universitätsklinikum Regensburg wurde in der zweiten Jahreshälfte 2012 aus einer bereits bestehenden Kooperation der Infektiologie, Mikrobiologie und Apotheke ein ABS-Programm aufgebaut und durch den Vorstand etabliert. Ein Universitätsklinikum mit seinen spezifischen Eigenschaften, Expertisen und Res- 
sourcen bietet dabei besondere Herausforderungen (z. B. viele schwerkranke, immunsupprimierte Patienten und ein breites Fächerspektrum). Es bietet aber auch gute Gelegenheiten für begleitende wissenschaftliche Evaluationen und für die Integration des Programms in die Aus-, Weiter- und Fortbildung. Das Einbeziehen von Doktoranden, die an spezifischen wissenschaftlichen Projekten arbeiten, und spezielle Lehrvisiten bedeuten eine direkte Verbesserung und Intensivierung der wissenschaftlichen und klinischen Ausbildung in diesem wichtigen Aspekt der Medizin. Im Folgenden werden wir die Erfahrungen mit diesem Programm darstellen.

\section{Struktur und Team}

Die Bildung eines geeigneten Teams und dessen Etablieren durch die Klinikleitung sind wichtige Eckpunkte eines ABS-Programms. Idealerweise sollte ein ABSTeam aus klinischen Infektiologen, klinischen Mikrobiologen bzw. Krankenhaushygienikern und Pharmazeuten bestehen. Die speziellen Expertisen dieser Gruppen sind wichtig in der Beratung und für das Monitoring der Ergebnisse sowie für das Feedback an die direkt therapierenden Ärzte.

Eine Unterstützung der Krankenhausleitung ist essenziell-sowohl in der expliziten Übertragung von Aufgaben für das ABS-Team als auch organisatorisch, z.B. durch hinreichende Personalressourcen. Die Legitimation des Teams durch den Klinikumsvorstand ist Voraussetzung für eine erfolgreiche Arbeit, sie sichert allein aber noch nicht die unerlässliche Akzeptanz des Teams in der täglichen Arbeit auf den Normal- und Intensivstationen.

\section{FAZIT FÜR DIE PRAXIS}

Die Akzeptanz des ABS-Teams auf den Stationen ist der wichtigste Faktor für die erfolgreiche Einführung der notwendigen Maßnahmen. Sie setzt eine gute Teamstruktur und gegenseitige Vertretungsmöglichkeiten für mehrere Teilexpertisen voraus.

Das ABS-Team am Universitätsklinikum Regensburg bestand von Beginn an aus klinischen Infektiologen, Mikrobiologen bzw. Krankenhaushygienikern und Apothekern. Die 4 Teammitglieder der ersten Stunde hatten sämtlich 4 ABS-Kurse absolviert und waren deshalb und durch die vorherige Zusammenarbeit schnell ein funktionierendes Team. Das Team ist weitergewachsen und hat derzeit 12 Mitglieder, die so eine sinnvolle Arbeitsteilung und gegenseitige Vertretung garantieren. Regelmäßige Besprechungen sind in der gemeinsamen Fallbesprechung der Mikrobiologie und Infektiologie unter ständiger Beteiligung der Klinikumsapotheke 1mal wöchentlich integriert.

\section{Arbeitsprogramm und Maßnahmen}

\section{Lokale Leitlinien erstellen und überarbeiten}

Die Kerngruppe aus Infektiologen, Mikrobiologen und Apothekern hatte bereits eine langjährige Erfahrung im Erarbeiten von lokalen Leitlinien zur Antibiotikatherapie. Diese wurden jeweils mit den Fachkliniken für alle Indikationen seit 1999 erstellt und im Arzneimittelleitfaden des UK Regensburg veröffentlicht. Diese Arbeit wurde mit der Etablierung des ABS-Programms in dieses integriert, und man vereinbarte regelmäßige Überarbeitungen und Anpassungen der Leitlinien sowie eine neue Veröffentlichung im klinikeigenen Informationssystem. Neben dem therapeutischen Einsatz von antimikrobiellen Substanzen werden in der aktuellen Phase der Überarbeitung auch erstmalig die Prophylaxen integriert (u. a. perioperativ, bei Immunsuppression).

\section{ABS-Visiten}

Visiten auf allen Intensivstationen des Klinikums (internistische, allgemeinchirurgische, herz-thoraxchirurgische und neurochirurgische Intensivstationen) finden wöchentlich statt. Zusätzlich gibt es eine wöchentlich rotierende Visite auf den derzeit 4 hämatologischen Stationen mit einer hohen Rate an neutropenischen bzw. stammzelltransplantierten Patienten. Andere Stationen werden entweder auf Anforderung oder bei häufigen Problemen mit Infektionen in die Visiten einbezogen.

\section{TIPP FÜR DIE PRAXIS}

Der Fokus der Visiten ist in erster Linie die klinische Beratung bei einzelnen Patienten. Problemfälle werden gemeinsam diskutiert, diagnostische Verfahren und Methoden kritisch diskutiert und auch relevante und potenzielle Interaktionen aufgezeigt. Diese Visiten erfordern einen hohen Aufwand, auch in der Vorbereitung.

\section{Restriktion von Reserveantibiotika}

Eine Liste von Antiinfektiva ist in der Apotheke als Reserveantibiotikum gekennzeichnet, hierzu gehören derzeit:
- Linezolid
- Daptomycin
- Tigecyclin
- einige Antimykotika

Sobald diese Substanzen von einer Station angefordert werden, wird ein Konsil des ABS-Teams generiert, das dann prüft, ob der Einsatz sinnvoll oder gar nötig ist. Damit die Therapiesicherheit gewährleistet wird, ist der initiale Einsatz dieser Substanzen bis zu einer Therapiedauer von $72 \mathrm{~h}$ möglich, z.B. am Wochenende. 
Für eine längere Belieferung der Station ist dann das Vorliegen eines Konsils nötig. Ausgenommen von dieser Regelung sind Patientengruppen mit einem besonderen Krankheitsspektrum, z. B. neutropenische Patienten, bei denen ein häufiger empirischer Einsatz von Antimykotika in allen Leitlinien praktisch verankert ist.

\section{Selektiver Bericht zur Empfindlichkeit von Antibiotika}

Viele Labore berichten aus eingesandten Materialien gefundene Erreger und deren Antibiotikaempfindlichkeiten und -resistenzen für alle getesteten Antibiotika (nicht selektiv). Die Interpretation mikrobiologischer Befunde muss prinzipiell in enger Kooperation zwischen Klinikern und Mikrobiologen erfolgen. Unkommentierte und nicht diskutierte Befunde führen beim unerfahrenen Arzt oft dazu, ein Antibiotikum auszuwählen, das alle nachgewiesenen Keime „abdeckt“, jedoch ohne die optimale Wirksamkeit in Betracht zu ziehen, z. B. beim Einsatz eines Chinolons bei einer Bakteriämie mit Enterokokken und ausgewiesener Empfindlichkeit ( $\mathbf{A b b}$. 2).

Selektive Berichte zur Antibiotikaempfindlichkeit lösen selbstverständlich nicht das Problem mangelnder Kommunikation (das häufig schon bei der Anforderung beginnt). Sie können aber dazu beitragen, gerade den unerfahrenen Benutzer nicht in die oben beschriebene Falle tappen zu lassen.

\section{Merke}

Beim selektiven Antibiogramm werden nur die für den entsprechenden Erreger und die Organinfektion geeigneten Antibiotika angegeben. Damit wird je-

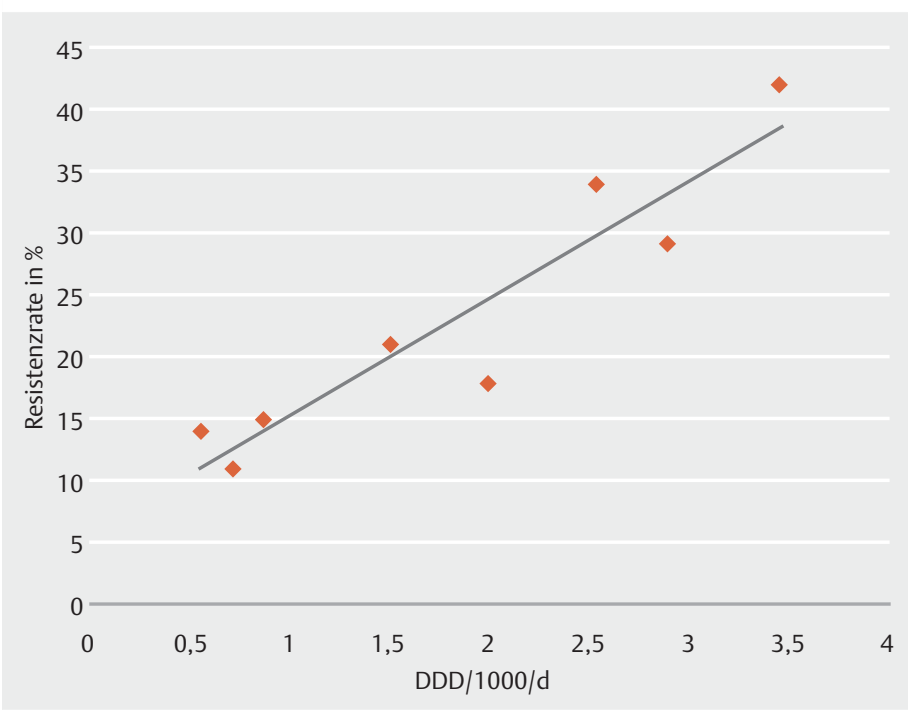

- Abb. 2 Korrelation von Chinolonresistenz und Chinoloneinsatz in der ambulanten Therapie von Infektionen in verschiedenen europäischen Ländern 2014, ECDC, EARS [1]. weils die erste Wahl der Antibiotikatherapie für die entsprechende Infektion fokussiert. Ein weiterer Aspekt ist die Steuerung des Antibiotikareinsatzes.

Bei Infektionen mit den meisten grampositiven Erregern werden z.B. auch Cephalosporine der 3. Generation als wirksam ausgewiesen. Die Nutzung des selektiven Antibiogramms kann hier steuernd einwirken und Reduktionen beim Einsatz dieser Gruppe erreichen.

Für das Erstellen selektiver Berichte zur Antibiotikaempfindlichkeit bzw. -resistenz wurden nach intensiver Literaturrecherche für verschiedene Erreger je nach Material (z.B. unterschiedlich für Hautabstriche und Blutkulturen) Algorithmen zur Ergebnismitteilung von Antibiotika im Institut für Mikrobiologie aufgestellt. Diese Algorithmen wurden auf ihre Kongruenz zu internationalen und nationalen Leitlinien geprüft, im ABSTeam diskutiert und dann in der Klinik eingeführt. Parallel untersuchte man den Zusammenhang zwischen mikrobiologischen Befunden und Antibiotikaverschreibungen. Für die erste Phase des Projekts erfolgten neben einer klinikumsweiten Fragebogenaktion Punktprävalenzuntersuchungen zur Antibiotikatherapie sowie Analysen zum Antibiotikaverbrauch am UK Regensburg. Als Intervention wurde nun der neue mikrobiologische Befund mit dem selektiven Antibiogramm (s.o.) gewählt. Danach untersuchte man prospektiv, ob durch diese Befundänderung ein verändertes Verschreibungsverhalten von Antibiotika zu messen ist.

\section{FAZIT FÜR DIE PRAXIS}

Die Akzeptanz der neuen Befunde auf den Normalstationen war von Anfang an gut, hier wird das schnelle Hinleiten zur optimalen Therapie geschätzt. Auf einigen Intensivstationen dagegen kritisierte man die Wahl der Antibiotika zunächst als zu eng. Durch Schulungen, besonders zur Ableitbarkeit für weitere Substanzen, und durch eine weitere Kommentierung der Befunde ließ sich eine deutlich höhere Akzeptanz erzielen.

\section{Fokussierte Maßnahmen}

\section{Behandlung der S.-aureus-Bakteriämie}

Eines der ersten Projekte fokussierte sich auf die Therapie von Staphylococcus-aureus-Bakteriämien. Dies sind schwer verlaufende Infektionen mit hoher Komplikationsrate und hoher Sterblichkeit. Aus vielen Untersuchungen ist bekannt, dass die Einhaltung diagnostischer und therapeutischer Standards das Behandlungsergebnis beeinflusst (u.a. Suche nach Absiedlungen, mindestens 14-tägige intravenöse Therapie mit einem Antibiotikum der ersten Wahl) [12]. Ein Nachweis von 
S. aureus in einer Blutkultur ist praktisch nie als Kontamination einzustufen, weshalb hier ein Monitoring der Behandlung durch Benachrichtigung aus dem mikrobiologischen Labor gut zu etablieren ist. Interventionen zur besseren Behandlung der S.-aureus-Bakteriämie wurde auch bereits in ABS-Programmen mit anderen Settings erfolgreich durchgeführt.

In einer Initialphase analysierte man retro- bzw. prospektiv über 24 Monate Qualitätsparameter der Behandlung. Als Intervention wurde ein Leitfaden erstellt, der bereits mit der ersten schriftlichen Befundübermittlung übersandt wird. Zusätzlich erfolgt ein Hinweis zur Anforderung eines klinischen Konsils zur Beratung. Die Veränderungen der Qualitätsparameter und des klinischen Erfolgs werden prospektiv evaluiert.

\section{Behandlung der ambulant erworbenen Pneumonie}

Die Behandlung der ambulant erworbenen Pneumonie erfolgt bereits unter Erfassung einer Reihe von Qualitätsindikatoren und auch des Behandlungserfolgs. Allerdings ist z. B. nicht klar, ob die klinische Einordnung des Schweregrads nach dem CRB-65-Risikoscores auf die Patientenkollektive in Universitätskliniken anwendbar ist-sowohl was die Indikation zur stationären Behandlung betrifft als auch in Bezug auf den Verlauf. Unter den Qualitätsindikatoren ist zwar der Zeitpunkt der Antibiotikatherapie, nicht aber die eigentliche Antibiotikatherapie und auch nicht die erfolgte mikrobiologische Diagnostik zu finden. Die benutzten Qualitätsindikatoren sind also gerade für die Frage der adäquaten Diagnostik und Therapie der Infektion nicht geeignet. Wir haben deshalb über einen mehrjährigen Zeitraum die stationär behandelten Fälle der ambulant erworbenen Pneumonie in Bezug auf die Patientencharakteristika, die mikrobiologische Diagnostik und die eingesetzten Antibiotika untersucht und werden nach den Ergebnissen entscheiden, ob hier Interventionen zur besseren Behandlung sinnvoll oder nötig sind.

Weitere in der Entwicklung befindlichen Projekte sind der empirische Antibiotikaeinsatz in der Notaufnahme des Klinikums für definierte Infektionen (u.a. Harnwegsinfektionen).

\section{Auswertung, Bewertung, Rückmeldung}

\section{Merke}

Die Aus- und Bewertung der erfolgten Maßnahmen ist ein wichtiger Teil bei ABS-Programmen. Sie zeigen einerseits Erfolg und Misserfolge der Maßnahmen, können helfen zukünftige Arbeitsfelder zu identifizieren, dienen andererseits aber auch der wichtigen Rückmeldung an die antibiotikaeinsetzenden Ärzte und an den Vorstand des Klinikums.
Für die Aus- und Bewertung und v. a. für die Rückmeldung der Maßnahmen sind Verbrauchsdaten zu Antibiotika wie auch die Entwicklung von Resistenzen sicherlich die einfachsten und handlichsten Instrumente. Das eigentliche Ziel, eine adäquate und optimale Antibiotikatherapie für jeden individuellen Patienten, ist nicht leicht zu messen - erst recht nicht über ein gesamtes Klinikum hinweg. Deshalb müssen zusätzlich andere Variablen als Surrogatmarker für die Qualität der Behandlung herangezogen und bewertet werden.

\section{Verbrauch}

Bereits durch das Infektionsschutzgesetz sind Krankenhäuser verpflichtet, ihren Antibiotikaverbrauch aufzuzeichnen und zu bewerten. Eine Möglichkeit hierzu wurde durch eine Kooperation der Universitätsklinik Freiburg und des Bundesverbands deutscher Krankenhausapotheker geschaffen, das ADKA-IF-Projekt. Mit dem Übersenden von Verbrauchsdaten und Pflegetagen wird damit ein übersichtlicher Bericht nach Stationen bzw. nach Abteilungen erstellt. Dieser Bericht lässt sich auch dazu nutzen, sich mit anderen Krankenhäusern der gleichen Größe zu vergleichen und auch spezifische Gruppen von Antiinfektiva in den Fokus zu nehmen.

Insgesamt konnten wir im Zeitraum nach Beginn des ABS-Programms bei gleichbleibendem steigendem Schweregrad der behandelten Patienten im Gesamteinsatz von Antibiotika eine Reduktion von ca. $10 \%$ beobachten ( $\$$ Tab. 1 ).

Positiv sieht die Bilanz auch bei den Reservesubstanzen aus: So konnte man z. B. den Einsatz von Linezolid um $60 \%$ reduzieren. Die Analyse zeigt jedoch auch Problemfelder auf: Im Vergleich zu anderen Kliniken der gleichen Größe werden sowohl Carbapeneme als auch Glykopeptide sehr häufig eingesetzt. Hier entwickelt man derzeit spezifische Interventionen.

\section{Resistenzentwicklung}

Resistenzentwicklungen werden aufgrund der Fallzahlen nur für das gesamte Klinikum analysiert und bewertet, die Statistiken 3-monatlich ausgewertet und mit den Trends der deutschen und europäischen Surveillance-Netzwerke verglichen. Hier fanden sich bisher

- Tab. 1 Antibiotika- und Antimykotikaverbrauch des Universitätsklinikums Regensburg 2012 - 2015 (in RDD/100 Patiententage; RDD: recommended daily dose, für jedes Antiinfektivum definierter Größe).

\begin{tabular}{|l|r|r|r|r|}
\hline Jahr & $\mathbf{2 0 1 2}$ & $\mathbf{2 0 1 3}$ & $\mathbf{2 0 1 4}$ & $\mathbf{2 0 1 5}$ \\
\hline Antibiotika & 62,5 & 57,6 & 53,1 & 53,8 \\
\hline Antimykotika & 9,1 & 8,6 & 8,4 & 9,1 \\
\hline Case Mix Index & 1.94 & 1.96 & 1.99 & 2.00 \\
\hline
\end{tabular}


keine deutlichen Abweichungen zu den Entwicklungen bei nationalen Resistenzraten bei grampositiven und gramnegativen Erregern.

\section{Einhalten der Antibiotikarestriktion}

Prinzipiell sind Belieferungen von Stationen mit restringierten Antibiotika ohne Zustimmung des ABS-Teams nicht möglich. Allerdings kann in begrenztem Maß durch Vorratsbildung diese Restriktion umgangen werden, auch trotz expliziten Einspruch der Klinikdirektoren. Die Adhärenz der Maßnahmen zur Restriktion von Antibiotika wurde anhand von Arzneimittelbestellungen auf den Stationen ausgewertet und in einer Stichprobe überprüft. Dabei stellte sich heraus, dass in $95 \%$ aller Fälle die Restriktionen befolgt und diesen nicht widersprochen wurde.

\section{Einfluss von $A B S-V i s i t e n$ auf Intensivstationen}

Der Abschluss der ABS-Fortbildung beinhaltet auch eine Projektarbeit. Zwei dieser Projektarbeiten haben die neu eingeführten ABS-Visiten auf den Intensivstationen untersucht. Beide Untersuchungen dauerten 2 Monate und es erfolgte jeweils ein Vergleich mit 2 Quartalen vor der Einführung. Auf einer internistischen Intensivstation wurden bei $58 \%$ aller Patienten Änderungen der Therapie vorgeschlagen. $42 \%$ betrafen Deeskalationen, 14\% ein Absetzen aufgrund der Therapiedauer. Die Anregungen wurden zu $98 \%$ umgesetzt. Parallel dazu erkannte man bei einer untersuchten chirurgischen Station ebenfalls hohe Interventionsraten: Bei $90 \%$ aller Patienten schlug man Interventionen vor, $91 \%$ wurden umgesetzt. Auf beiden Stationen fand sich bei gleicher Fallzahl eine Gesamtreduktion des Antibiotikaverbrauchs von ca. $14 \%$. Vor der Intervention war auf der chirurgischen Station ein aufällig hoher Verbrauch von Linezolid vorhanden (fallbezogen im Vergleich zu den anderen Intensivstationen), hier ließ sich der Verbrauch um $60 \%$ reduzieren.

\section{Qualitätsindikatoren}

Qualitätsindikatoren können Surrogatmarker für das schwer zu messende Ziel einer adäquaten Antibiotikatherapie sein. Bisher gibt es keinen konsentierten und evaluierten Satzvon Qualitätsindikatoren für hospitalisierte Patienten. Es gibt eine Reihe von Vorschlägen für solche Indikatoren, allerdings ist nur bei spezifischen Infektionen ein klarer Zusammenhang mit dem Behandlungsergebnis als härtestem Kriterium vorhanden.

\section{Merke}

Weiterhin muss ein Qualitätsindikator gut und zuverlässig zu messen sein, er muss Verbesserungspotenzial beinhalten und nach Möglichkeit auch für ein Benchmarking zur Verfügung stehen.
Zwei Gruppen haben solche generischen (nicht auf spezifische Infektionen bezogenen) Qualitätsindikatoren in einem Konsensverfahren beschrieben: In einer niederländischen Studie wurden 11, in einer deutschen Konsensstudie 42 solcher Indikatoren vorgeschlagen $[13,14]$. Mittlerweile sind die 11 Qualitätsindikatoren in einer Gruppe von niederländischen Krankenhäusern evaluiert worden ( Tab.2). Hier hatten z. B. alle Kliniken hauseigene Leitlinien, weshalb sich also kein Verbesserungspotenzial ergab. Andere Parameter, wie z. B. therapeutisches Drug-Monitoring, konnte zu selten angewandt werden (bei weniger als $10 \%$ aller Patienten), sodass dieser Parameter als Qualitätsindikator nicht sinnvoll ist.

\section{FAZIT FÜR DIE PRAXIS}

Gerade die Messbarkeit im klinischen Alltag und das mögliche Verbesserungspotenzial sind wichtig für die eigene Auswahl - diese Faktoren können zwischen verschiedenen Kliniken stark variieren [15].

Wir haben am Universitätsklinikum Regensburg bisher nur strukturelle Qualitätsindikatoren etabliert, z. B. die lokalen Leitlinien, die wiederum auf evidenzbasierten nationalen bzw. internationalen Leitlinien aufbauen. Prozessorientierte Indikatoren aus $>$ Tab.2, die man im nächsten Programm-Schritt integrieren kann, werden derzeit v.a. auf die kritischen Punkte der verlässlichen Messbarkeit und des Potenzials für Verbesserungen in unserem Umfeld geprüft.

\section{Zusammenfassung und Ausblick}

Am Universitätsklinikum Regensburg wurde im Jahr 2012 ein Antibiotic-Stewardship-Programm etabliert mit dem Ziel, dort die Qualität der Antiinfektivatherapie zu verbessern und damit langfristig den Trend der bakteriellen Resistenzentwicklung aufzuhalten. Das Team besteht aus klinischen Infektiologen, Mikrobiologen/Krankenhaushygienikern und Apothekern mit Expertise und einer intensiven Ausbildung in der Behandlung von Infektionskrankheiten und auch in den Prinzipien und Methoden von Antibiotic-Stewardship-Programmen.

Neben der Etablierung des Programms durch ABSTeam und Vorstand des Klinikums ist als zweites wichtiges Strukturelement eine langjährige Arbeit an lokalen Leitlinien zur Antibiotikatherapie hier bereits vorhanden gewesen. Diese Leitlinien wurden aktualisiert und werden derzeit erneut konsentiert. 
- Tab.2 Generelle Qualitätsindikatoren für die antimikrobielle Therapie [15].

\begin{tabular}{|c|c|c|c|c|}
\hline \multirow[t]{2}{*}{ Qualitätsindikatoren } & \multicolumn{4}{|c|}{ Bemerkungen } \\
\hline & $\begin{array}{l}\text { messbar bei } \\
>75 \%\end{array}$ & $\begin{array}{l}\text { anwendbar bei } \\
>10 \%\end{array}$ & $\begin{array}{l}\text { hohe Inter- } \\
\text { observer-Varia- } \\
\text { bilität }(k>0,4)\end{array}$ & $\begin{array}{l}\text { Verbesserungs- } \\
\text { potenzial }>15 \%\end{array}$ \\
\hline \multicolumn{5}{|l|}{ Strukturell } \\
\hline $\begin{array}{l}\text { Eine hauseigene Leitlinie zur Antibiotikatherapie soll vorhanden } \\
\text { sein und 3-jährlich aktualisiert werden. }\end{array}$ & Ja & Ja & $\mathrm{n} / \mathrm{a}$ & Nein \\
\hline $\begin{array}{l}\text { Die hauseigene Leitlinie soll auf internationalen Leitlinien basieren, } \\
\text { angepasst auf die lokale Resistenzsituation. }{ }^{*}\end{array}$ & Ja & Ja & $\mathrm{n} / \mathrm{a}$ & Ja \\
\hline \multicolumn{5}{|l|}{ Prozessbezogen } \\
\hline Antibiotikaeinsatz erfolgt leitliniengerecht. ${ }^{*}$ & Ja & Ja & Ja & Ja \\
\hline Abnahme von 2 Paaren Blutkulturflaschen vor Therapie* & Ja & Ja & Ja & Ja \\
\hline $\begin{array}{l}\text { Vor Antibiotikaeinsatz erfolgt Probenentnahme an vermuteten } \\
\text { Infektionsherden. }{ }^{1}\end{array}$ & Ja & Ja & Ja & Ja \\
\hline $\begin{array}{l}\text { Indikation und vorgesehene Dauer der Antibiotikatherapie soll } \\
\text { dokumentiert sein. }{ }^{1}\end{array}$ & Ja & Ja & Ja & Ja \\
\hline $\begin{array}{l}\text { Umstellung auf orale Antibiotikatherapie nach } 48-72 \mathrm{~h} \text { (nach } \\
\text { klinischem Verlauf und falls durchführbar). }{ }^{*}\end{array}$ & Ja & Ja & Ja & Ja \\
\hline Fokussierung der Therapie nach der Erregeridentifizierung. ${ }^{*}$ & Ja & Ja & $\mathrm{Ja}$ & $\mathrm{Ja}$ \\
\hline $\begin{array}{l}\text { Dosis und Intervall der Dosierung soll an die Nierenfunktion } \\
\text { adaptiert werden. }\end{array}$ & $\mathrm{Ja}$ & Nein & $\mathrm{Ja}$ & $\mathrm{Ja}$ \\
\hline $\begin{array}{l}\text { Therapeutisches Drug-Monitoring sollte bei Aminoglykosiden } \\
\text { bei Therapiedauer }>3 \mathrm{~d} \text { und } \\
\text { Vancomycin bei Therapiedauer }>5 \mathrm{~d} \text { erfolgen. }\end{array}$ & $\mathrm{Ja}$ & Nein & $\mathrm{Ja}$ & \\
\hline $\begin{array}{l}\text { Empirisch begonnene Antibiotikatherapien sollten bei fehlendem } \\
\text { klinischen oder mikrobiologischem Hinweis für eine bakterielle } \\
\text { Infektion abgesetzt werden. } \\
\text { Das Maximum der Therapiedauer einer empirischen Therapie } \\
\text { sollte } 7 \mathrm{~d} \text { sein. }\end{array}$ & $\mathrm{Ja}$ & Nein & Nein & \\
\hline
\end{tabular}

Im Fokus der Arbeit lag zunächst explizit die individuelle klinische Beratung bei Problempatienten, wobei immer wieder adäquate Diagnostik und Therapie diskutiert und die Grundsätze besprochen wurden. Das explizite Ziel dieser Arbeit war, durch punktuelle Beratung mit hoher medizinischer Expertise neben der individuellen Fallbehandlung den Prozess der Antibiotikatherapie in den besuchten Einrichtungen positiv zu beeinflussen. Die Akzeptanz dieser Visiten ist hoch, zu erkennen auch an der parallel sich positiv entwickelnden Bilanz der Anforderungen an die beteiligten Gruppen. Die Leistungszahlen der mikrobiologischen Diagnostik, der patientenspezifischen infektiologischen Konsile und auch der pharmakologischen Beratung (z.B. zu Arzneimittelinteraktionen) sind deutlich und stetig angestiegen.

Als Ergebnis der bisherigen Arbeit konnte man den Einsatz von Antibiotika und Antiinfektiva um insgesamt ca. $10 \%$ reduzieren. Auf den spezifisch besuchten Sta- tionen werden die vorgeschlagenen Änderungen der Therapie weitestgehend umgesetzt. Dort zeigen sich pointierte Änderungen im Verbrauch von Antiinfektiva - auch in Bezug auf den Einsatz spezifischer Klassen.

Änderungen Antibiotikaverbrauch, wie wir sie auch gesehen haben, gelten aber generell als leicht zu erntende Früchte von ABS-Programmen, die sich nicht kontinuierlich fortsetzen lassen. Hier ist natürlich keine ständig weitergehende Reduktion zu erwarten, möglicherweise ist ein Plateau bereits erreicht. Beim Antibiotikaverbrauch muss in der Zukunft das Augenmerk auf spezifische Substanzen bzw. Substanzgruppen liegen hier kann ein Vergleich zu anderen Kliniken hilfreich sein.

Für die weitere Verbesserung der Antibiotikatherapie wird die Aus-, Weiter- und Fortbildung intensiviert, die Ausbildung zu Antibiotic Stewardship ist bereits in den Studentenunterricht integriert und auch spezifische lo- 
kale Fortbildungen sind eingeführt. Für die Verwirklichung weiterer Qualitätsverbesserungen werden derzeit geeignete Qualitätsindikatoren gesichtet, die von der Diagnostik über die erste empirische Therapie bis hin zur Entscheidung über das Therapieende den Prozess der Antibiotikatherapie adäquat abbilden. Je nach den Verbesserungspotenzialen in den einzelnen Bereichen nimmt man diese dann auch präferenziell spezifisch in den Fokus der weiteren Arbeit. Die je nach Station sinnvollen Indikatoren werden dann regelmäßig evaluiert und auch zur Rückkopplung benutzt.

\section{KERNAUSSAGEN}

- Die rasche Zunahme von Antibiotikaresistenzen ist ein weltweites Problem und droht viele Fortschritte der Medizin zunichte zu machen.

- Resistenzentwicklung und Antibiotikaeinsatz sind untrennbar miteinander verbunden - sowohl im Einsatz beim Menschen als auch bei Tieren.

- Antibiotic Stewardship ist ein Ansatz, der Optimierung der individuellen Therapie mit einem verantwortlichen Einsatz von Antibiotika im Hinblick auf die Resistenzentwicklung verbindet

- ABS benötigt die Unterstützung der Klinikleitung und definierte Ressourcen.

- ABS sollte in Teamarbeit zwischen Infektiologen, Mikrobiologen / Krankenhaushygienikern und Pharmazeuten erfolgen.

- ABS-Programme verbessern erfolgreich die Qualität der Antibiotikatherapie und wirken der Resistenzentwicklung entgegen.

- ABS sollte in deutschen Krankenhäusern breit etabliert werden.

Interessenkonflikte

Die Autoren geben an, dass kein Interessenkonflikt besteht.

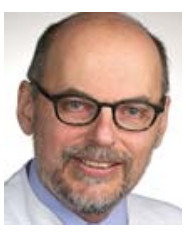

\section{Bernd Salzberger}

Prof. Dr.med., Facharzt für Innere Medizin und Infektiologe, leitet die Stabstelle Infektiologie und das ABS-Programm des Universitätsklinikums Regensburg. $\mathrm{Er}$ wurde nach klinischer und Forschungstätigkeit in Köln und Seattle 2001 auf die Professur für klinische Infektiologie an der Universität Regensburg berufen. Spezialgebiete sind Antibiotikatherapie, Infektionen bei immunsupprimierten Patienten und klinische Virologie. Er ist im Vorstand der Deutschen Gesellschaft für Infektiologie.

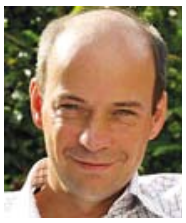

\section{André Gessner}

Prof. Dr. med., Dr. rer. nat., 1980-1986 Studium der Medizin an der Universität Hamburg, anschließend Studium der Molekularbiologie von 1986-1988. 1997 erfolgte die Habilitation an der FriedrichAlexander-Universität Erlangen-Nürnberg, 2001 Forschungstätigkeit an der University of California, San Francisco, USA, dann war er 2003-2010 stellv. Institutsleitung der Klinischen Mikrobiologie an der FriedrichAlexander-Universität Erlangen-Nürnberg. 2010 W3-Professur und Institutsleitung der Medizinischen Mikrobiologie und Hygiene an der Universität Regensburg. Seit 2015 ist er Forschungsdekan an der Medizinischen Fakultät der Universität Regensburg.

\section{Korrespondenzadresse}

Prof. Dr. med. Bernd Salzberger

Stabstelle Infektiologie

Universitätsklinikum Regensburg

Franz-Josef-Strauss Allee 11

93053 Regensburg

E-Mail: Bernd.Salzberger@ukr.de

Literatur

[1] European Centre for Disease Prevention and Control (ECDC). Surveillance Atlas of Infectious Diseases. European Antibiotic Resistance Surveillance. Im Internet: 2014: http://ecdc.europa.eu/en/healthtopics/antimicrobial-resistance-and-consumption/antimicrobial_resistance/database/Pages/database.aspx (Stand: 13.2.2017)

[2] Paul-Ehrlich-Gesellschaft für Chemotherapie e.V. Arbeitsgemeinschaft „Empfindlichkeitsprüfung und Resistenz“. Resistenzdaten. 2016: Im Internet: www.p-e-g.org/econtext/resistenzdaten (Stand: 13.2.107)

[3] The White House. National Action Plan for Combating Antibiotic-Resistant Bacteria. 2015: Im Internet: www.cdc.gov/ drugresistance/pdf/national_action_plan_for_combating_antibotic-resistant_bacteria.pdf (Stand: 13.2.107)

[4] New York Times. Penicillin Finder Assays its Future. New York Times 1945: 21 
[5] Carlet ], Collignon P, Goldmann D et al. Society's failure to protect a precious resource: antibiotics. The Lancet 2011; 378: $369-371$

[6] Kollef MH, Micek ST. Rational use of antibiotics in the ICU: balancing stewardship and clinical outcomes. JAMA 2014; 312: $1403-1404$

[7] Barlam TF, Cosgrove SE, Abbo LM et al. Implementing an Antibiotic Stewardship Program: Guidelines by the Infectious Diseases Society of America and the Society for Healthcare Epidemiology of America. Clin Infect Dis 2016; 62: e51-e77

[8] Pollack LA, van Santen KL, Weiner LM et al. Antibiotic Stewardship Programs in U.S. Acute Care Hospitals: Findings From the 2014 National Healthcare Safety Network Annual Hospital Survey. Clin Infect Dis 2016; 63: 443 - 449

[9] Bundesministerum für Gesundheit, Bundesministerium für Ernährung und Landwirtschaft, Bundesministerium für Bildung und Forschung. Deutsche Antibiotika-Resistenzstrategie (DART 2020): Antibiotika-Resistenzen bekämpfen zum Wohl von Mensch und Tier. Beschluss des Bundeskabinetts vom 13.5.2015. Im Internet: http://www.bundesgesundheitsministerium.de/fileadmin/Dateien/Publikationen/Ministerium/Broschueren/BMG_DART_2020_Bericht_dt.pdf (Stand: 13.2.107)

[10] Centers for Disease Control and Prevention (CDC). Core Elements of Hospital Antibiotic Stewardship Programs. Atlanta: GA: US Department of Health and Human Services; CDC; 2014: Im Internet: www.cdc.gov/getsmart/healthcare/pdfs/core-elements.pdf (Stand: 13.2.107)
[11] de With K, Allerberger F, Amann S et al. Strategies to enhance rational use of antibiotics in hospital: a guideline by the German Society for Infectious Diseases. Infection 2016; 44: $395-439$

[12] Bai AD, Showler A, Burry L et al. Impact of Infectious Disease Consultation on Quality of Care, Mortality, and Length of Stay in Staphylococcus aureus Bacteremia: Results From a Large Multicenter Cohort Study. Clin Infect Dis 2015; 60: $1451-1461$

[13] Thern J, de With K, Strauss R et al. Selection of hospital antimicrobial prescribing quality indicators: a consensus among German antibiotic stewardship (ABS) networkers. Infection 2014; 42: 351 - 362

[14] van den Bosch CM, Geerlings SE, Natsch S et al. Quality indicators to measure appropriate antibiotic use in hospitalized adults. Clin Infect Dis 2015; 60: 281 - 291

[15] van den Bosch CM, Hulscher ME, Natsch S et al. Applicability of generic quality indicators for appropriate antibiotic use in daily hospital practice: a cross-sectional point-prevalence multicenter study. Clin Microbiol Infect 2016; 22: 888.e1 888.e9

Bibliografie

DOI http://dx.doi.org/10.1055/s-0043-103659 Krankenhaushygiene up2date 2017; 12: 75-85 (c) Georg Thieme Verlag KG Stuttgart · New York ISSN 1862-5797 


\section{Punkte sammeln auf CME.thieme.de}

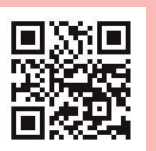

Diese Fortbildungseinheit ist 12 Monate online für die Teilnahme verfügbar.

Sollten Sie Fragen zur Online-Teilnahme haben, finden Sie unter http://cme.thieme.de/hilfe eine ausführliche Anleitung. Wir wünschen viel Erfolg beim Beantworten

der Fragen!

Unter eref.thieme.de/ZZX8MPK oder über den QR-Code kommen Sie direkt zum Artikel zur Eingabe der Antworten.

VNR 2760512017152374700

\section{Frage 1}

Welche Aussage zur Antibiotikaresistenz in Deutschland ist zutreffend?

A Die Rate der Methicillin-Resistenz bei S. aureus (MRSARate) liegt seit vielen Jahren über $50 \%$.

B Die Rate von Chinolonresistenzen bei E. coli ist konstant unter $15 \%$.

c Die Rate von E. coli, die Extended-Spektrum $\beta$-Laktamasen (ESBL) produzieren, ist mittlerweile bei mehr als $10 \%$.

D Die Rate der Vancomycin-Resistenz bei E. faecium liegt unter $5 \%$.

E Mehr als $70 \%$ aller E.-coli-Stämme sind empfindlich gegenüber Ampicillin.

\section{Frage 2}

Welche Aussage zu gesetzlichen Vorgaben und öffentlichen Unterstützung zur Bekämpfung von Antibiotikaresistenzen ist richtig?

A In den USA wird von den Centers for Disease Control (CDC) ein ABS-Programm für alle Krankenhäuser empfohlen.

B In Deutschland wird nach DART 2020 ein ABS-Programm für jedes Krankenhaus empfohlen.

c Eine Aufzeichnung von Resistenzraten im Krankenhaus ist in Deutschland nicht gesetzlich vorgesehen.

D Eine Aufzeichnung von Antibiotikaverbrauchsdaten in Krankenhäusern ist in Deutschland nicht gesetzlich vorgesehen.

E Die nationale und internationale Politik hat sich des Problems der Antibiotikaresistenz bisher gar nicht gestellt.

\section{Frage 3}

Welcher der folgenden Punkte ist nicht Kernelement eines Antibiotic-Stewardship-Programms nach Auffassung der CDC?

A Leadership Commitment (Commitment und Unterstützung der Krankenhausleitung)

B Tracking (Monitoring des Antibiotikaeinsatzes)

C Reporting (Rückmeldung der Verbrauchs- und Resistenzdaten)

D Education (regelmässige Fortbildung)

E Rewarding (Belohnung bei Reduktion des Einsatzes)

\section{Frage 4}

Welche Aussagen trifft für den Begriff „Antibiotic Stewardship“ zu?

A Da es eine griffige deutsche Übersetzung gibt, sollte dieser Begriff im Deutschen nicht mehr gebraucht werden.

B Stewardship bezieht sich auf die Aufgaben eines Schiffoder Flugstewards, dessen oberste Aufgabe ein guter Service ist.

c Eine gute Übersetzung für Steward ist „Steuermann“, dies beschreibt auch das oberste Ziel eines ABS-Programms.

D Als wichtigstes Element von Stewardship wird die Übernahme von Verantwortung angesehen.

E Antibiotic Stewardship wurde als Begriff erst mit der ersten deutschen Leitlinie 2014 eingeführt.

\section{Frage 5}

Wie ist der Stand von Antibiotic-Stewardship-Programmen bzw. -Maßnahmen in Deutschland?

A Nach dem Infektionsschutzgesetz soll jedes Krankenhaus ein ABS-Programm aufweisen.

B Die Aus- und Weiterbildung in Antibiotic Stewardship ist seit vielen Jahren Bestandteil der Weiterbildung in der Inneren Medizin und in der Mikrobiologie.

c In Deutschland gibt es bisher ca. 500 ABS-Experten mit einem Netzwerk, das auf der gemeinsamen Ausbildung basiert.

D Eine Fort- und Weiterbildung in Antibiotic Stewardship ist in Deutschland noch nicht möglich.

E In der Approbationsordnung für Medizinstudenten ist Antibiotic Stewardship bereits als eigenes Fach vorhanden.

\section{Frage 6}

Welche der folgenden Berufsgruppen gehört nicht in erster Linie zu einem ABS-Team?

A klinische Infektiologen

B Qualitätsmanager

C Krankenhausapotheker

D Krankenhaushygieniker

E klinische Mikrobiologen

Weitere Fragen auf der folgenden Seite ... 


\section{Punkte sammeln auf CME.thieme.de}

Fortsetzung ...

\section{Frage 7}

Welche der folgenden strukturellen Voraussetzungen wird als Qualitätsindikator häufig genannt?

A Vorhandensein einer aktuellen, an die lokale Resistenzsituation angepasste Leitlinie zur Antibiotikatherapie

B Vorhandensein eines hygienebeauftragten Arztes

C Vorhandensein einer Hygienekommission

D etabliertes Monitoring des Antibiotikaeinsatzes

E Unterstützung durch die Krankenhausleitung

\section{Frage 8}

Welcher der folgenden Qualitätsindikatoren ist für Veränderungen im Prozess der Antibiotikatherapie am ehesten geeignet?

A ein etabliertes ABS-Team, in dem alle Disziplinen vertreten sind

B eine lokale Leitlinie zur Antibiotikatherapie, die auf internationalen Leitlinien beruht

c die Abnahme einer Blutkultur vor Beginn einer Antibiotikatherapie

D ein Routinescreening bei Trachealsekreten und Urin auf Intensivstationen

E die Rate von Antibiotikatherapien, bei denen Indikation und Zeitdauer der geplanten Therapie dokumentiert sind

\section{Frage 9}

Welches der Ergebnisse von ABS-Programmen lässt sich i. d. R. rasch erreichen und gilt nicht als eigentliches Ziel?

A Reduktion von verlängerten Prophylaxetherapien ohne Indikation

B Reduktion des Gesamtverbrauchs und der Kosten

C Verbesserung der Adhärenz zu internationalen Leitlinien

D adäquate Dosierung bei Patienten mit Niereninsuffizienz

E Fokussierung der Therapie nach Erhalt von mikrobiologischen Befunden

\section{Frage 10}

Was bedeutet ein „selektives Antibiogramm“?

A selektive Mitteilung über besonders preisgünstige Antibiotika an Assistenzärzte

B selektive Mitteilung über Antibiotika zum Einsatz auf Normal- vs. Intensivstationen

c selektive Mitteilung über wirksame Antibiotika ohne Bewertung der klinischen Wirksamkeit

D selektive Mitteilung über Antibiotika, die zur Therapie nach nachgewiesenem Erreger und Infektionsort primär geeignet sind

E selektive Mitteilung über Antibiotikaempfindlichkeit an Ober- und Chefärzte, damit sie die Therapie steuern 